\title{
Procedure of computation and automatic marking of drill hole spacing at excavation of horizontal workings
}

\author{
Maxim K ononenko*, Ruslan Ganiev, and Roman K uzminykh \\ U ral State M ining University, 620144, 30, Kuibyshev st., Ekaterinburg, R ussia
}

\begin{abstract}
Contemporary approaches and techniques impacting subsurface penetration performance and penetration rate are studied and analyzed. Two main dimensions are identified, and the works performed during operation were compared. A solution based on implementation of modular architecture of the system with optional connection of modules, performing computations according to various techniques, enabling comparison of calculation results and selection of the most effective option, is proposed. A computational experiment based on real-objects data was conducted. The factors effecting drilling-and-blasting operations were identified during its course. A model designing the optical projections over targeted drill holes is also obtained. The proposed approach is more effective and accurate than manual application of drill hole spacing by paint; in addition, is saves a lot of time required for setting-up. A nother highlighted advantage of the developed approach is high quality and accuracy at marking of the drill hole spacing for blasting pattern in accordance with geometrical features of the surfaces, vs. approaches and techniques used at preparation to drilling earlier. A side from that, a clear trend of consistent improvement and perfection by means of application of intelligent algorithm is observed in this scope.
\end{abstract}

\section{Introduction}

Today, most of the mining companies conducting subsurface mining operations use drillingand-blasting for construction of level and incline working. Herewith, penetration performance significantly depends on accuracy of blasting pattern calculation and accuracy of spacing, drilling and planting of explosives, eventually. Quality of blasting subsequently effects further process stages, as well.

Thus, a company can suffer major economic losses as a result of poor quality of drillingand-blasting operations.

\footnotetext{
${ }^{*}$ Corresponding author: maxkononenko111@gmail.com
} 


\section{Materials and methods}

Two dimensions effecting penetration performance and penetration rate most significantly can be identified: first, this is accuracy and conformity to the actual specifics of generated blasting pattern, and secondly, human factor at spacing and blasthole drilling. The latter can be mitigated by using robotized drilling units capable of accomplishing the above operations without human participation (e.g., Sandvik DD 422IE or A tlas Copco B oomer), however the cost of such units is quite high, and their application is not al ways expedient.

As regards the first scope, there are a lot of approaches to estimation of the blasting pattern, each having its advantages and disadvantages. M ost of estimation techniques include empirical equations, containing some factors with pretty wide range of values, whose choice completely depends on experience of an expert calculating the parameters.

For example, one of the common techniques used for calculation of number of holes at cutting with angling holes suggests that design values listed in the Table 1 [1, p.20-21] are used.

Table 1. N umber of holes depending on rock hardness and sectional area of a working.

\begin{tabular}{|c|l|c|c|c|c|c|c|}
\hline \multirow{2}{*}{$\begin{array}{l}\text { Rock hardness } \\
\text { ratio }\end{array}$} & \multicolumn{5}{|c|}{ Number of holes per drilled depth depending on sectional area of a working, $\mathrm{m}^{2}$} \\
\cline { 2 - 8 } & 4 & 6 & 8 & 10 & 12 & 14 & 16 \\
\hline $2-4$ & $8-11$ & $12-16$ & $17-21$ & $22-27$ & $28-33$ & $34-38$ & $35-42$ \\
\hline $5-7$ & $12-16$ & $17-21$ & $22-27$ & $28-33$ & $34-38$ & $39-42$ & $43-46$ \\
\hline $8-10$ & $16-20$ & $21-26$ & $27-32$ & $33-37$ & $38-42$ & $42-46$ & $47-50$ \\
\hline $12-14$ & $20-24$ & $26-30$ & $32-36$ & $37-42$ & $42-46$ & $46-50$ & $50-54$ \\
\hline More than 14 & $26-28$ & $30-36$ & $36-40$ & $44-48$ & $48-52$ & $52-54$ & $56-60$ \\
\hline
\end{tabular}

A s the Table 1 shows, resulting values are not accurate numbers, e.g., with rock hardness 5 according to scale of M.M. Protodyakonov and rock section of a working $10 \mathrm{~m} 2$, the number of required holes ranges from 28 to 33. In fact, the estimations based on such approach, will have an averaged nature.

A part of physical and mechanical properties of the rock and specifications of explosive, technical specifications of the drilling units available at the company also impose their limitations on drill chart to be selected, therefore, selection of hole drill chart and estimation of blasting pattern present a multiple-factor problem, whose solution should be approached, taking into account all available information about external environment of the working.

Contemporary approaches to computation of blasting pattern make provision for dividing the holes according to their purpose into cutting, cropper and intermediate holes (for instance, refer to [2-4]). A pproaches as per such division are based on estimation of shear zone and fracture zone radii in accordance with the theory of rock blasting developed by B.N. K utuzov [5] with further plotting of drillhole spacing using these radii for calculation of spacing between blastholes (refer to Figure 1).

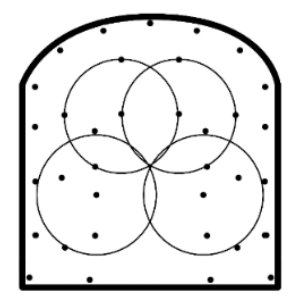

Fig. 1. A pproach to drillhole spacing configuration based on radii 
In accordance with [2-4], this approach results in saving of explosives and hole number reduction. However, no comprehensive comparison of hole spacing calculation methods using the same input data was conducted, since a very detailed (from the physics perspective) simulation model of blasting must be designed for this purpose, so it makes no sense to discuss significant advantages of one calculation approach over another for the time being.

To eliminate the human factor from procedures of generation and plotting of the drillhole spacing at actual site, the authors are developing a software-hardware system of automatic hole drilling with an option of automatic calculation and mapping drillhole spacing. The concept of this system is based on principles of laser scanning and optical guidance, and makes provision for installation on drilling units al ready existing at the plant with capability of transmission of signals to control units by means of servodrives. Conceptual flow chart of the unit for mapping the drillhole spacing is presented on Figure 2.

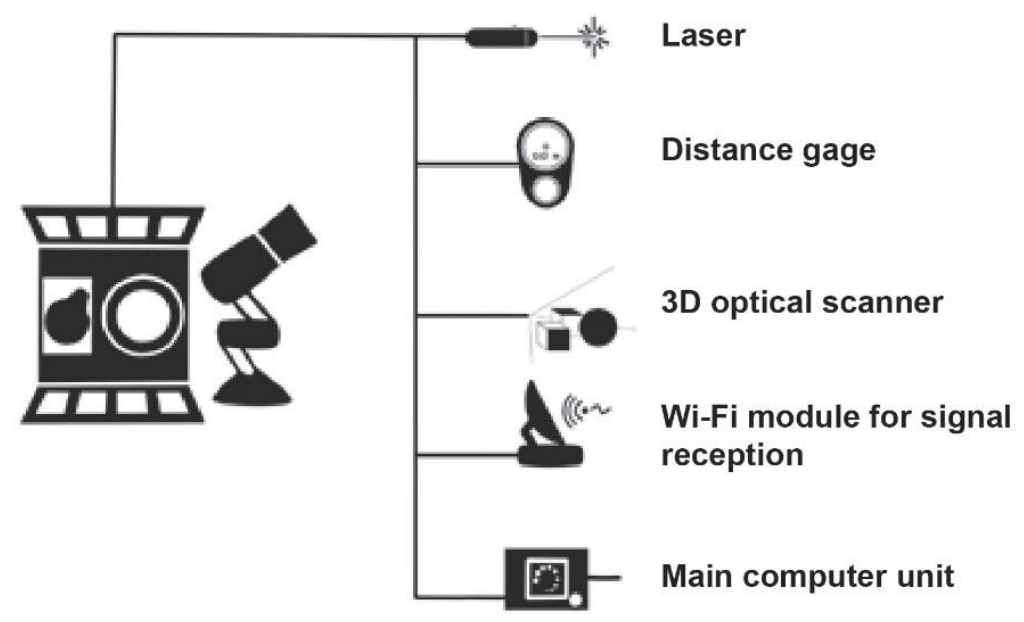

Fig. 2. Conceptual flow chart of drillhole spacing mapping system

B ased on the above analysis of existing approaches to cal culations of blasting pattern, the authors of this paper made a decision to use modular architecture of the system with optional connection of modules, performing computations according to various techniques, enabling comparison of calculation results and selection of the most effective option. A rchitecture of the software implementation of solution of this problem is presented on Figure 3 in form of A rchi $M$ ate design diagram.

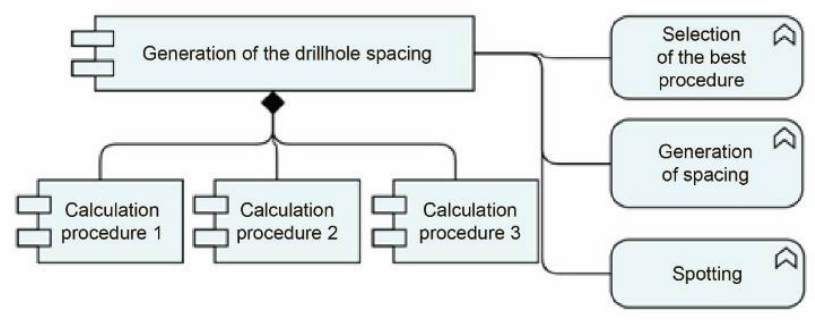

Fig. 3. A rchitecture of drillhole spacing generation and spotting subsystem 
Figure 3 displays three drillhole spacing computation procedures as the modules, comprising the system, and selection of the best procedure, generation of spacing and spotting are highlighted as the key nontrivial functions of the system. Selection of the best calculation procedure initiates calculation according to all procedures added to the system as modules, and provides comparative table listing number of drillholes, weight of required explosives and scope of drilling operations. The function of generation of spacing initiates laser scanning of the working, after which it imposes generated hole drill chart over the model of actual site. The spotting function converts the drill chart in a set of control commands of the servodrive, guiding the laser to required position for the following highlight of drilling locations.

The project utilized such cutting-edge technologies as multiagent approach ([5]), neural networks and intelligent algorithms ([6]) abundantly used for modelling, prediction and control of the processes in mining. These technologies can also be used for modelling and controlling the hole drilling at excavation of horizontal workings. For example, the best computation procedure can be selected, or a new procedure can be designed through neural network, based on existing ones.

$B$ ased on international experience related to location of potential damage zones in tunnel blasting operations, according to M WD (measure while drilling) data ([7]) during simulation experiment based on real site data, some factors resulting in error at drilling the holes as per design were identified:

- The bearing surface can differ considerably from that specified in the certificate, which prevents the input from being pre-configured with high accuracy

- The drill hole shape is not an ideal geometrical figure, which results in errors of area estimation

- The very rock surface prepared for drilling-and-blasting operations is not an ideal plane, so the optical model overlaying error must be taken into consideration during actual generation of drillhole spacing

After taking the above factors into account, the data from some sites were entered and analysis was conducted with input in conformity with blasting pattern. As a result, we obtained a model of imposing optical projection on projected bottom holes.

\section{Results and discussion}

Highlighting the drilling location with laser is a more effective method than manual application of drillhole spacing by means of paint (such an approach, described in [8], for example, was used by many companies), which is most commonly used when fully automatic drilling units are not operated.

In addition, it can be observed that the capabilities of data mining, computer vision and laser scanning not only enable generation of the most accurate drillhole spacing in accordance with geometrical properties of the surface, but increase accuracy of drilling itself.

\section{Conclusions}

In the long run, it is planned to design a simulation model that would enable testing the computation results according to various procedures and to identify the best option, based on intelligent algorithm, which would increase accuracy of calculations and total ly eliminate the human factor, since engagement of an expert for selection of the best approach to designing the drillhole spacing would become reduntant. 


\section{Acknowledgement}

The research paper is part of the study funded in terms of the grant by UM NIK, Contract N 0 . 14207G U/2019 dated 21.06.2019

\section{References}

1. M.V. Kornilkov, N.N. Leshchukov, A.G. Petrushin, Design and calculation of the blasting pattern for horizontal workings, 43 (Ekaterinburg: The Ural State M ining University Press, 2010)

2. S.A. Vokhmin, G.S. Kurchin, A.K. K irsanov, P.A. Deryagin, Herald of the M oscow State Technical University named after G.I. Nosov, 4(48) (2014)

3. A.K. K irsanov, S.A. Vokhmin, G.S. K urchin, J ournal of Siberian Federal U niversity. Engineering and Technology, 4, 396-405 (2015)

4. G.G. Linvinsky, P.N. Shulgin, News; The State U niversity of Tula. Earth Sciences, 1, $90-97(2012)$

5. B.N. K utuzov, N ew theory and cutting-edge technologies of destruction of the minerals by linear charges of explosives, 95 (Novosibirst: Science, 2002)

5. E.A. V olkova, A.V. D ruzhinin, Y u.A. Lagunova, V.N. Poluzadov, The mining research and information bulletin, 11(37), 103-111 (2019) doi: 10.25018/0236-1493-2019-1137-103-111

6. E.A. Volkova, New refractories, 4 (2018)

7. J. Navarroa, J.A.Sanchidriána, P. Segarraa, R. Castedoa, E. Costamagna, M. López, Tunnelling and Underground Space Technology, 504-516 (2018)

8. K.G. Karginov, A.I. Lipovoy, T.M. Teziev, D.V. Ryabov, The mining research and information bulletin, 8 (2001) 